\title{
Vacunar de influenza a los trabajadores de salud: tiempo para la ética y el profesionalismo
}

\section{Healthcare workers influeza vaccination: it is a time for ethics and professionalism}

\author{
Ignacio Marín-León* \\ Departamento de Medicina Interna, Fundación Enebro, CIBERESP-IBIS, Hospital Virgen del Rocío, Sevilla, España
}

La gripe estacional es una infección vírica aguda que se propaga fácilmente entre las personas, principalmente por gotas de la tos. De los tres tipos de virus influenza, el tipo A es el de mayor circulación en todo el mundo, más proclive a mutaciones antigénicas y el que cursa con mayor gravedad. La influenza provocada por el virus puede ser grave en niños pequeños y adultos mayores 0 en personas diagnosticadas con diabetes, obesidad mórbida, problemas renales o con defensas bajas, y se puede complicar por infecciones secundarias por bacterias, que pueden ocasionar fallecimientos ${ }^{1}$.

Cada año se producen hasta 650,000 defunciones por enfermedades respiratorias relacionadas con la gripe estacional, según nuevas estimaciones de la Organización Mundial de la Salud (OMS). La mayoría de las defunciones se producen entre personas de 75 o más años y en niños menores de 5 años que se integran en los sectores más desfavorecidos y menos protegidos de la sociedad 1 .

Así, en la temporada 2018-2019, para los EE.UU., el Centro para el Control y Prevención de Enfermedades calculó que se produjeron entre 37.4-42.9 millones de enfermos con influenza, 17.3-20.1 millones de consultas médicas, 531,000-647,000 de ingresos relacionados y entre 36,000-61,200 casos de muerte por influenza ${ }^{2}$. Estas cifras indican la elevada carga de morbilidad por gripe y su sustancial costo social y económico para el mundo, asimismo destacan la importancia que tiene la prevención de las epidemias estacionales de gripe ${ }^{3}$.

A pesar de la disponibilidad de fármacos antivirales, la vacunación es la estrategia más efectiva y eficiente contra la enfermedad. Nuevas tecnologías en la elaboración de vacunas han mejorado la tolerabilidad, facilidad de uso, eficacia y capacidad de protección en orden a facilitar la cobertura de vacunación tanto en el ámbito poblacional como de los trabajadores de la salud $(\mathrm{TdS})^{4}$. Por ello, antes de que se produzca la próxima pandemia, se requiere un fortalecimiento de los sistemas de salud con el fin de mejorar la salud de las personas más vulnerables y más expuestas a riesgos.

La OMS alienta a los países a que otorguen prioridad a la prevención de la gripe, particularmente para las personas expuestas a alto riesgo de complicaciones graves y defunción por gripe, y para los profesionales del sector sanitario'.

Debido a su exposición a los pacientes, los profesionales sanitarios corren gran riesgo de infectarse por los virus de la gripe y de transmitirlos. Los TdS en riesgo incluyen a los de centros, servicios y establecimientos sanitarios, tanto de atención primaria como especializada y hospitalaria, pública y privada, así como personal de oficinas de farmacia. Aunque el concepto de TdS es variable entre países y dentro
Correspondencia:

*Ignacio Marín-León

E-mail: gpcfabry@gmail.com

1405-9622/( 2019 Sociedad Médico-Quirúrgica del Hospital Juárez de México, A.C. Publicado por Permanyer. Este es un artículo open access bajo la licencia CC BY-NC-ND (http://creativecommons.org/licenses/by-nc-nd/4.0/).
Disponible en internet: 10-03-2020 Rev Hosp Jua Mex. 2020;87(1):2-5 www.revistahospitaljuarez.com 
de contextos organizativos, los datos de incidencia de gripe en este heterogéneo colectivo según la revisión sistemática realizada por Lietz ${ }^{5}$, que incluye 26 estudios y 1 metaanálisis de 15 estudios sobre riesgo profesional de influenza entre TdS en la epidemia de 2009, encuentra una prevalencia combinada del $6.3 \%$ de gripe estacional, con el doble de riesgo de sufrir gripe que la población general de la misma edad.

No obstante, a pesar de las recomendaciones sobre inmunización de dicho grupo de profesionales, se sigue detectando una deficiente cobertura en los últimos años, que dista mucho de la recomendación mínima (más del 90\% de apego a la vacunación). La cobertura de vacunación entre TdS varía entre países, siendo del $30 \%$ en Europa a pesar de las campañas institucionales de los Sistemas Nacionales de Salud (SNS), y siendo más alta en EE.UU., Canadá, Australia, Japón y Arabia Saudí. Faltan datos en muchos países, incluido todo el continente americano al sur del Río Grande ${ }^{6}$. En México, los datos disponibles presentan cierta inconsistencia, mientras que en 2018 se observó que la vacunación contra la influenza en trabajadores del Instituto Nacional de Ciencias Médicas y Nutrición Salvador Zubirán rondaba el $50 \%$ en promedio ${ }^{7}$, los datos de cobertura aportados a la Organización Panamericana de Salud (OPS) por México en 2018 son de un 94\% de los adultos mayores y para los TdS del $100 \%{ }^{8}$. Por su parte, la ENCOVAN reporta una cobertura del $56.13 \%$ para adultos mayores de 60 años en 2008 en los Estados Unidos Mexicanos ${ }^{9}$.

¿A qué se debe tan baja cobertura entre los profesionales del sector salud? Según una reciente revisión sistemática, el principal determinante de la aceptación de la vacunación por TdS incluye el deseo de protegerse a sí mismo y a su familia, más que el riesgo absoluto de enfermedad. Es interesante constatar que la motivación de autoprotegerse es el doble que el deseo de proteger a sus pacientes o de mantener íntegro el SNS ${ }^{10}$. Además, la resistencia a la vacunación contra la gripe estacional entre los TdS se explica entre otros factores por la baja percepción de riesgo, la negación del valor social de la inmunización, la baja presión social, no haber sufrido gripes o no haberse vacunado previamente y la dificultad de acceso a la vacunación ${ }^{11}$.

Hay disponibles cuatro estudios que muestran que la intención favorable a vacunarse era del $22.83 \%$, y cumplimentan la vacunación el $13.53 \%$. Se muestra que la actitud favorable la condicionan la mayor titulación y conocimientos, el deseo de protegerse y recibir información o la proposición de vacunarse por vía institucional. Ser enfermera o TdS auxiliar disminuye la tasa de vacunación ${ }^{12}$.

Una revisión de 17 estudios encuentra que la adherencia a la vacunación de la gripe varía entre $<10$ al $53 \%$, y los determinantes de una mayor adherencia son la utilización de estrategias multifactoriales de promoción ${ }^{13}$.

En otra revisión de 20 estudios que incluían a más de 22,300 TdS, se encuentra una cobertura de entre el 9 y el $29 \%$, y los determinantes para no vacunarse son el no convivir con más familiares y las dudas sobre la seguridad y la efectividad de la vacuna, así como la creencia de la influencia de la industria farmacéutica en la decisión de promover la vacunación ${ }^{14}$.

Estos datos son relevantes para diseñar estrategias de implantación de programas que aumenten la cobertura entre los TdS.

Un análisis de esta situación y percepciones sitúa los principios del profesionalismo en el centro del escenario para reflexionar sobre cómo se promueve la vacunación contra el virus de la influenza/gripe de los profesionales de la salud en la campaña 2019-2020.

La carta del profesionalismo médico y las organizaciones sanitarias propone al menos tres ejes del mismo ${ }^{15}$ :

- Enfocarse en los pacientes: compromiso para preocuparse de lo que es importante para ellos.

- Cultura de organización: compromiso para el bienestar de las personas, la construcción de equipos de trabajo y un ambiente saludable.

- Enfocarse a la comunidad: compromiso para enfocar los determinantes de la salud, los mejores valores comunitarios y su mayor beneficio.

Respecto a la responsabilidad con los pacientes, esta es bidireccional, tanto para evitar ser la persona que puede trasmitir la gripe a aquellas que tienen un alto riesgo de padecer complicaciones, como de contagiarse de gripe en los momentos en que el recurso del personal sanitario es más necesario por la situación de epidemia.

Algunos profesionales argumentan que los estudios no han demostrado un efecto en la trasmisión de gripe a los pacientes, ni un efecto de la vacunación de los TdS en evitarlo, tal como parece plausible biológicamente. Quizá la falta de confirmación del efecto vacunación se deba a que precisamente el TdS que no se vacuna y sufre la gripe falta al trabajo y evita contagios $^{5}$. 
Hay, además, el factor de ejemplaridad social y sobre la formación de los futuros profesionales, pues parte de la responsabilidad de la educación médica es formar profesionales competentes para mejorar la salud de la población ${ }^{16}$.

Respecto a la responsabilidad con el sistema de salud y la institución, es relevante resaltar que la vacunación poblacional evita ingresos, gastos y la disminución de productividad social. Se ha observado que el costo de la vacunación contra la influenza supone solo el gasto aproximado de entre el 23 y el $27 \%$ de lo que ahorra, y además romper la cadena de contagio elimina gastos de bolsillo en salud ${ }^{17}$.

Por su demostrada eficacia en proteger a los TdS sanos y jóvenes, así como en evitar días de ausencia laboral por enfermedad, la vacunación es un elemento de mejora del clima laboral y del compañerismo, al evitar sobrecargar la asistencia sobre los TdS que se vacunan, por faltar al trabajo los que enferman durante la epidemia por no estar vacunados. Por ello, están en debate las propuestas de vacunación imperativa para los profesionales ${ }^{18}$.

Aunque es sabido que el profesionalismo puede no ser suficiente para alcanzar los profundos cambios que necesitan los sistemas de salud y que sin un hondo sentido profesional los sistemas de salud están perdidos $^{19}$, en términos prácticos, la cuestión a responder es ¿cómo conseguir que los TdS acepten ser vacunados anualmente contra la epidemia de influenza?

Los ensayos clínicos que han testado estrategias de implantación de la vacunación entre TdS, muestran un mayor éxito con estrategias combinadas de educación e incentivación con medidas favorecedoras, y que incluyen también intervenciones mandatorias. Así se confirma en una revisión sistemática que encuentra que los principales condicionantes de estrategias efectivas para incrementar la vacunación de los profesionales es una combinación de varios factores, tales como promover una actitud positiva sobre la eficacia y seguridad de la vacunación y sobre la percepción de beneficio para ellos y otros, así como cumplir con normas sociales y profesionales $^{20}$.

No obstante, hay controversia sobre la legalidad de imponer de modo mandatorio la obligación de vacunarse a los profesionales, dado la ausencia de evidencias sobre el efecto en los pacientes ${ }^{5}$, aunque la ética del nuevo profesionalismo debe dejar pocas dudas respecto a la obligación moral de vacunarse. Estamos en tiempos para la acción, ya que tales controversias no son razón suficiente que deban frenar los programas que impliquen a los trabajadores del sector salud en la protección de los pacientes, de las organizaciones sanitarias y de ellos mismos y sus compañeros.

\section{Conflicto de intereses}

No se declaran conflictos de intereses.

\section{Financiamiento}

No se recibió financiación.

\section{Responsabilidades éticas}

Protección de personas y animales. Los autores declaran que para esta investigación no se han realizado experimentos en seres humanos ni en animales.

Confidencialidad de los datos. Los autores declaran que han seguido los protocolos de su centro de trabajo sobre la publicación de datos de pacientes.

Derecho a la privacidad y consentimiento informado. Los autores han obtenido el consentimiento informado de los pacientes y/o sujetos referidos en el artículo. Este documento obra en poder del autor de correspondencia.

\section{Bibliografía}

1. World Health Organization (WHO). Influenza (seasonal). Fact sheet November 2016. Disponible en: WWW.who.int

2. https://espanol.cdc.gov/enes/flu/about/burden/2018-2019.html

3. Nair $H$, Abdullah Brooks W, Katz MA, Roca A, Berkley JA, Madhi SA, et al. Global burden of respiratory infections due to seasonal influenza in young children: a systematic review and meta-analysis. Lancet. 2011;378:1917-30.

4. Ansaldi $F$, Durando $P$, Icardi $G$. Intradermal influenza vaccine and new devices: a promising chance for vaccine improvement. Expert Opin Biol Ther. 2011;11(3):415-27.

5. Lietz J, Westermann C, Nienhaus A, Schablon A. The Occupational Risk of Influenza A (H1N1) Infection among Healthcare Personnel during the 2009 Pandemic: A Systematic Review and Meta-Analysis of Observational Studies. PLoS One. 2016;11(8):e0162061.

6. To KW, Lai A, Lee KC, Koh D, Lee SS. Increasing the coverage of influenza vaccination in healthcare workers: review of challenges and solutions. J Hosp Infect. 2016;94(2):133-42.

7. Instituto Nacional de Ciencias Médicas y Nutrición Salvador Zubirán. Vacunación contra influenza. Información básica. Disponible en: http:// www.innsz.mx/2018/Carrusel/Campanas/VACUNACION2018.pdf

8. Organización Panamericana de Salud. Inmunización: Datos mapas y estadísticas. http://ais.paho.org/imm/influenzaCoverageMap.asp

9. Trejo Valvidia B, Mendoza Alvarado LR, Palma Coca O, Hernández Ávila M, Téllez-Rojo Solís M. Encuesta Nacional de Cobertura de Vacunación (influenza, neumococo y tétanos) en Adultos Mayores de 60 años en México. Salud Pública de Mex. 2012;54(1):39-46.

10. La Torre G, Mannocci A, Ursillo P, Bontempi C, Firenze A, Panico MG, et al. Prevalence of influenza vaccination among nurses and ancillary workers in Italy: systematic review and meta analysis. Hum Vaccin. 2011;7(7):728-33.

11. Dini G, Toletone A, Sticchi L, Orsi A, Bragazzi NL, Durando P. Influenza vaccination in healthcare workers: A comprehensive critical appraisal of the literature. Hum Vaccin Immunother. 2018;14(3):772-89. 
12. Bish A, Yardley L, Nicoll A, Michie S. Factors associated with uptake of vaccination against pandemic influenza: a systematic review. Vaccine. 2011;29(38):6472-84.

13. Maggiore UL, Scala C, Toletone A, Debarbieri N, Perria M, D'Amico, et al. Susceptibility to vaccine-preventable diseases and vaccination adherence among healthcare workers in Italy: A cross-sectional survey at a regional acute-care university hospital and a systematic review. Hum Vaccin Immunother. 2017:13(2):470-6.

14. Prematunge C, Corace K, McCarthy A, Nair RC, Pugsley R, Garber G. Factors influencing pandemic influenza vaccination of healthcare workers-a systematic review. Vaccine. 2012;30(32):4733-43.

15. ABIM Foundation. American Board of Internal Medicine; ACP-ASIM Foundation. American College of Physicians- American Society of Internal Medicine; European Federation of Internal Medicine. Medical professionalism in the new millennium: A physician charter. Ann Intern Med. 2002;136(2):243-6.
16. Fajardo Dolci GE, Santacruz Varela J, Lara Padilla E, García Luna Martínez E, Zermeño Guerra A, et al. Características generales de la educación médica en México. Una mirada desde las escuelas de medicina. Salud Publica Mex. 2019;61(5):648-56.

17. Gutiérrez JP, Bertozzi SM. Vacunación contra influenza para adultos mayores en México: consideraciones económicas. Salud Publica Mex. 2005;47(3):234-9.

18. Bodenheimer T, Sinsky C. From triple to quadruple aim: Care of the patient requires care of the provider. Ann Fam Med. 2014;12(6):573-6.

19. Jacobs R, Mannion R, Davies HT, Harrison S, Konteh F, Walshe K. The relationship between organizational culture and performance in acute hospitals. Soc Sci Med. 2013;76(1):115-25.

20. Corace KM, Srigley JA, Hargadon DP, Yu D, MacDonald TK, Fabrigar LR, et al. Using behavior change frameworks to improve healthcare worke influenza vaccination rates: A systematic review. Vaccine. 2016; 34(28):3235-42. 